\title{
Snail maintains metastatic potential, cancer stem- like properties, and chemoresistance in mesenchymal mouse breast cancer TUBO-P2J cells
}

\author{
SUN YOUNG MA ${ }^{1}$, JIN-HEE PARK ${ }^{2}$, HANA JUNG ${ }^{2}$, SUNG-MIN HA ${ }^{2}$, YEONYE KIM ${ }^{2}$, \\ DONG HYEN PARK ${ }^{3}$, DEUK HEE LEE ${ }^{3}$, SOOYONG LEE ${ }^{1}$, IN-HO CHU ${ }^{4}$, \\ SO YOUNG JUNG ${ }^{5}$, IL-HWAN KIM ${ }^{6}$, IL-WHAN CHOI ${ }^{2}$, \\ CHANG SOO $\mathrm{CHOI}^{4}$ and SAEGWANG PARK ${ }^{2}$

\footnotetext{
${ }^{1}$ Department of Radiation Oncology, Kosin University College of Medicine, Busan 49267;

${ }^{2}$ Department of Microbiology and Immunology, INJE University College of Medicine;

${ }^{4}$ Department of Surgery, INJE University College of Medicine; ${ }^{5}$ Department of Dermatology,

Haeundae Paik Hospital, INJE University College of Medicine, Busan 47392;

${ }^{6}$ Department of Internal Medicine Division of Hemato-Oncology, Heaundea Paik Hospital,

INJE University College of Medicine, Busan 48108, Republic of Korea
} \\ ${ }^{3}$ Department of Radiation Oncology, Busan-Paik Hospital, INJE University College of Medicine;
}

Received December 5, 2016; Accepted July 10, 2017

DOI: 10.3892/or.2017.5834

\begin{abstract}
Snail, a zinc-finger transcriptional repressor of E-cadherin expression, is one of the key inducers of epithelial-mesenchymal transition (EMT) in epithelial cancer. In breast cancer, EMT has been associated with malignancies, including metastasis, cancer stem-like properties, and resistance to chemotherapy and radiotherapy. In this study, we analysed the role of Snail in the highly metastatic mesenchymal TUBO-P2J mouse breast cancer cells, by loss of function using short hairpin RNA. Though silencing Snail did not restore the E-cadherin expression or induce morphological changes, Snail silencing significantly ablated in vitro and in vivo metastatic potentials. In addition, Snail silencing also reduced resistance to chemotherapy drugs and cancer stem-like properties, such as CD44 expression, aldehyde dehydrogenase (ALDH) activity, colony formation, and in vivo tumour formation and growth. However, radioresistance was not decreased by silencing Snail. Collectively, this study suggested that Snail is a main regulator of the maintenance of malignancy potentials and is a good target to prevent cancer metastasis and to increase chemotherapy susceptibility.
\end{abstract}

Correspondence to: Professor SaeGwang Park, Department of Microbiology and Immunology, INJE University College of Medicine, 75 Bokji-ro, Busanjin-gu, Busan 47392, Republic of Korea

E-mail: micpsg@inje.ac.kr

Key words: breast cancer, Snail, metastasis, cancer stem cell, chemoresistance, radioresistance

\section{Introduction}

Epithelial-mesenchymal transition (EMT) is a process where cells lose epithelial phenotypes, decrease cell-cell recognition and adhesion, and gain mesenchymal phenotypes and an increased potential for metastasis (1). The EMT process is essential in embryonic and breast development. In tumour progression and metastasis, EMT processes are also important. Cells with a mesenchymal phenotype show an increased potential of migration and invasion, anoikis resistance, chemoresistance, radioresistance, and stemness (1-3). E-cadherin loss is considered a hallmark of EMT. In the EMT process, the expression of E-cadherin is controlled by transcriptional and post-transcriptional regulation. E-cadherin gene (CDH1) expression is modulated by several transcription repressors, which are typically expressed in mesenchymal cells, including Snail genes, Twist, Zeb genes and E47 $(1,4)$. The zinc-finger transcriptional repressor Snail is one of the genes frequently associated with EMT $(4,5)$. Previous studies indicated that Snail may participate in the progression of breast cancer and other types of cancer via the downregulation of CDH1 and the upregulation of mesenchymal genes (4). It was also reported that Snail participates in metastasis, cancer stemness, recurrence, and resistance to chemotherapy and radiotherapy (6-9).

In a previous study, we reported on the TUBO-P2J spontaneous metastatic mouse breast cancer cell line, which is derived from TUBO, a non-metastatic epithelial breast cancer cell line (10). The TUBO-P2J cell line showed a mesenchymal phenotype compared to TUBO, including a loss of E-cadherin, downregulation of claudin 1 and occludin 1 , and a gain of vimentin, $\alpha$-sm-actin and fibronectin. Various EMT transcription factors (EMT-TF), including Snail, Twist, FoxC2 and ZEB2, are also upregulated in TUBO-P2J cells. In addition, 
TUBO-P2J cells showed increased resistance to chemotherapy and radiotherapy $(10,11)$. However, it is not clear which factors are critical in the resistance to chemotherapy and radiotherapy.

In this study, we attempted to define the role of Snail in TUBO-P2J cells in the maintenance of mesenchymal cell phenotypes, including the loss of E-cadherin, metastasis, stemness, and resistance to chemotherapy and radiotherapy through a knock-down of Snail with short hairpin RNA (shRNA). Our results showed that Snail is not essential in the maintenance of mesenchymal cell phenotypes, such as cell morphology, the loss of E-cadherin, and resistance to radiation, but is essential for metastasis, stemness and resistance to chemotherapy. In addition, E-cadherin transcription was recovered by DNA methyltransferase (DNMT) inhibitor (5-aza-2'-deoxycytidine, 5 -aza-dC) alone, but protein expression was detected only in Snail knock-down cells treated with both 5 -aza-dC and an HDAC inhibitor (SAHA).

\section{Materials and methods}

Cell culture, shRNA construct and chemicals. The TUBO and TUBO-P2J cell lines were cultured in vitro in high glucose DMEM (HyClone ${ }^{\mathrm{TM}}$; Thermo Fisher Scientific, MA, USA) supplemented with heat-inactivated $10 \%$ foetal bovine serum (FBS; Thermo Fisher Scientific) and 1\% penicillin-streptomycin (HyClone; Thermo Fisher Scientific); the cells were maintained under $5 \% \mathrm{CO}_{2}$. Snail shRNA constructs targeting the Mus musculus sequences of Snai1 (NM_011427) were purchased from Sigma (Sh1-sequence: GTACCGGATGTGTC TCCCAGAACTATTTCTCGAGAAATAGTTCTGGGAGAC ACATTTTTTTG, Sh3-sequence: CCGG GATCTTCAACTG CAAATATTGCTCGAGCAATATTTGCAGTTGAAGATCT TTTTG). 5-Aza-dC (DNA methyltransferase inhibitor), SAHA (HDAC inhibitor), epirubicin and doxorubicin was purchased from Sigma.

Generation of Snail knockdown stable cell lines. The lentiviral transduction particles are produced from a library of sequenceverified lentiviral plasmid vectors for mouse snail gene (Sigma). Transduction of lentiviral particles were performed according to the manufacturer's instructions. Transduced cells were selected with $500 \mu \mathrm{g} / \mathrm{ml}$ of puromycin for 3-14 days. Purimycin-resistant colonies and separated single cells were picked. Each single cell was expanded to evaluate the knockdown status of the Snail gene via PCR and western blotting.

Inhibition of DNA methyltransferase and/or histone deacetylase. Cells $\left(1 \times 10^{6} / \mathrm{dish}\right)$ were seeded on $10 \mathrm{~cm}$ culture plate and incubated for $5 \mathrm{~h}$ for the cells to attach to the dishes. 5-Aza-dc $(2.5 \mu \mathrm{M})$ and/or SAHA $(0.5$ or $2 \mu \mathrm{M})$ were treated and incubated at $37^{\circ} \mathrm{C}$ in a humid atmosphere of $5 \% \mathrm{CO}_{2}$ for 3 or 7 days, then cells were subcultured with trypsinization to prevent overgrowth.

Reverse transcription-PCR. Total RNA extracted from cultured cells was used as a template for reverse transcriptase reactions. Aliquots of cDNA were amplified using the mouse and human primer pairs (Table I). After an initial denaturation at $94^{\circ} \mathrm{C}$ for $5 \mathrm{~min}$, the following was performed: 30 cycles of denaturation at $94^{\circ} \mathrm{C}$ for $30 \mathrm{sec}$, annealing at $55-60^{\circ} \mathrm{C}$ for
$30 \mathrm{sec}$, and extension at $72^{\circ} \mathrm{C}$ for $30 \mathrm{sec}$. PCR was performed with a 2720 thermo cycler (Applied Biosystems, CA, USA). The reaction products were analysed in $1.5 \%$ agarose gels.

Western blot assay. Cell lysates (40 $\mu \mathrm{g} / \mathrm{lane})$ were electrophoresed on polyacrylamide-SDS gels and then transferred to polyvinylidene fluoride membranes. Immunoblotting was performed by primary antibodies. E-cadherin, Snail-1 and GAPDH antibodies were obtained from Cell Signaling Technology (MA, USA) and estrogen receptor $\alpha$ (ESR1) antibody was purchased from Santa Cruz (TX, USA). HRP-conjugated secondary antibodies were purchased from Jackson Laboratories (Jackson ImmunoResearch, PA, USA).

Flow cytometry. To detect CD24 and CD44 expression, $2 \times 10^{5}$ cells were stained with $0.5 \mu \mathrm{g} / \mathrm{ml}$ of PE-Cy5-conjugated CD24 and FITC-conjugated CD44 antibody (BioLegend) for $30 \mathrm{~min}$ at room temperature in the dark. For aldehyde dehydrogenase (ALDH) activity, cells were stained using the Aldefluor kit (Stem Cell Technologies Inc., Vancouver, Canada) according to the manufacturer's protocol. Stained cells were acquired with the BD FACSCanto II cytometry system and analysed with FlowJo software (version 10).

Migration and invasion assays. The migration and invasion assays were performed using $8.0-\mu \mathrm{m}$ pore size 24 -well insert systems (BD Falcon) with $2 \mathrm{mg} / \mathrm{ml}$ of Matrigel coating (invasion) or not (migration). Then, $5 \times 10^{4}$ cells (migration) or $5 \times 10^{5}$ cells (invasion) were added to the upper chamber and incubated for 4-6 h (migration) or $72 \mathrm{~h}$ (invasion). After incubation, the upper surface of the membrane was wiped with a cotton-tipped applicator to remove residual cells. Cells in the bottom compartment were fixed and stained with H\&E. Cells in ten randomly selected fields at $\times 40$ magnification were counted.

Soft agar colony-forming assay. Six-well plates were covered with a layer of $0.5 \%$ agar in medium supplemented with $10 \%$ foetal bovine serum. Cells were prepared in $0.3 \%$ agar and seeded in triplicate at 3 different dilutions ranging from $1 \times 10^{3}$ to $5 \times 10^{5}$. The plates were incubated at $37^{\circ} \mathrm{C}$ in a humid atmosphere of $5 \% \mathrm{CO}_{2}$ for 2 weeks. Each experiment was repeated at least 3 times. Colonies were photographed between 10 and 12 days at an original magnification of $x 40$ under phase contrast.

Chemo-susceptibility test. The chemo-susceptibility was measured with an In Vitro Toxicology assay kit (TOX6, Sigma). Briefly, $0.5-1 \times 10^{4}$ cells/well were seeded and attached in 96-well culture plates. Indicated doses of chemo-drugs were administered for $72 \mathrm{~h}$. Cells were fixed in $10 \%$ trichloroacetic acid for $1 \mathrm{~h}$ at $4^{\circ} \mathrm{C}$, stained with SRB for $15 \mathrm{~min}$, and washed 3 times with $1 \%$ acetic acid. The incorporated dye was solubilized with $10 \mathrm{mM}$ Tris base, $\mathrm{pH}$ 8.8. Absorbance was spectrophotometrically measured at $565 \mathrm{~nm}$ using an EL800 microplate reader (Bio-Tek Instruments, Winooski, VT, USA).

Radiation susceptibility test. Cells were harvested with trypsin and suspended in $15 \mathrm{ml}$ conical tubes at $1 \times 10^{4}$ cells $/ \mathrm{ml}$. After irradiation with the indicated doses with $\mathrm{X}$-ray generator 
Table I. PCR primer pairs of mouse and human genes.

\begin{tabular}{llllll}
\hline Genes & Species & NCBI no. & \multicolumn{1}{c}{ Forward (5'-3') } & \multicolumn{2}{c}{ Reverse (5'-3') } \\
\hline CDH1 & Human & NM_004360 & ATTTTTCCCTCGACACCCGAT & TCCCAGGCGTAGACCAAGA \\
Snail & Human & NM_005985 & TCGGAAGCCTAACTACAGCGA & AGATGAGCATTGGCAGCGAG & 140 \\
GAPDH & Human & NM_001256799 & GGAGCGAGATCCCTCCAAAAT & GGCTGTTGTCATACTTCTCATGG & 197 \\
CDH1 & Mouse & NM_009864.2 & CCATTTTCACGCGCGCTG & CGCGAGCTTGAGATGGAT & 396 \\
DDR2 & Mouse & NM_022563.2 & ATCACAGCCTCAAGTCAGTGG & TTCAGGTCATCGGGTTGCAC & 116 \\
Snail & Mouse & NM_011427.2 & CACACGCTGCCTTGTGTCT & GGTCAGCAAAAGCACGGTT & 133 \\
Snail2 & Mouse & NM_011415.2 & ATGCCCAGTCTAGGAAATCG & TGATGACAACCAGGCATCAT & 551 \\
Foxc2 & Mouse & NM_013519.2 & AACCCAACAGCAAACTTTCCC & GCGTAGCTCGATAGGGCAG & 130 \\
Vimentin & Mouse & NM_011701.4 & CGGCTGCGAGAGAAATTGC & CCACTTTCCGTTCAAGGTCAAG & 124 \\
Fibronectin & Mouse & NM_010233.1 & AGAGCAAGCCTGAGCCTGAAG & TCGCCAATCTTGTAGGACTGACC & 192 \\
Twist & Mouse & NM_011658.2 & GGACAAGCTGAGCAAGATTCA & CGGAGAAGGCGTAGCTGAG & 146 \\
GAPDH & Mouse & NM_008084.2 & TTCACCACCATGGAGAAGGC & GGCATGGACTGTGGTCATGA & 250 \\
\hline
\end{tabular}

A

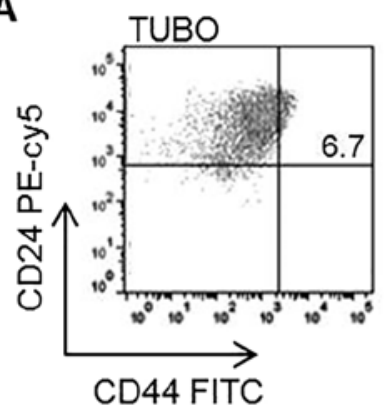

C

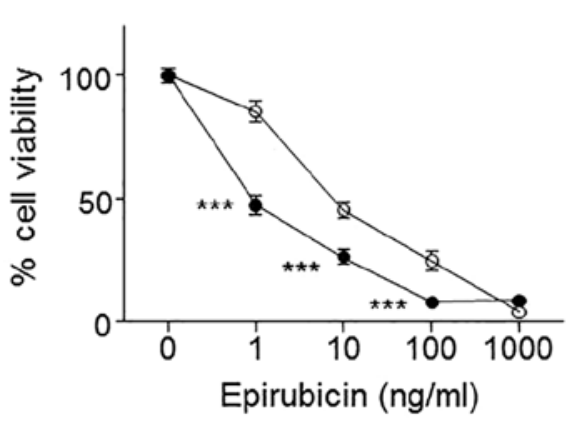

B

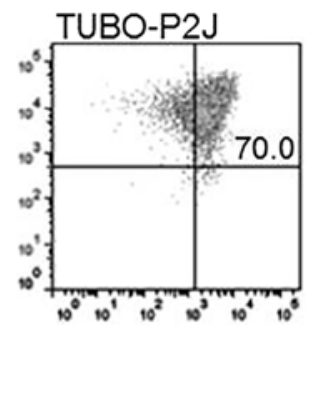

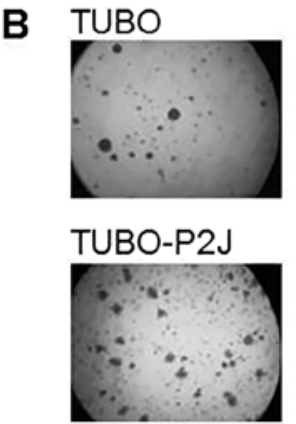

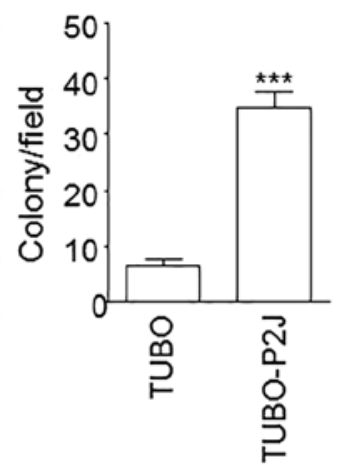

D
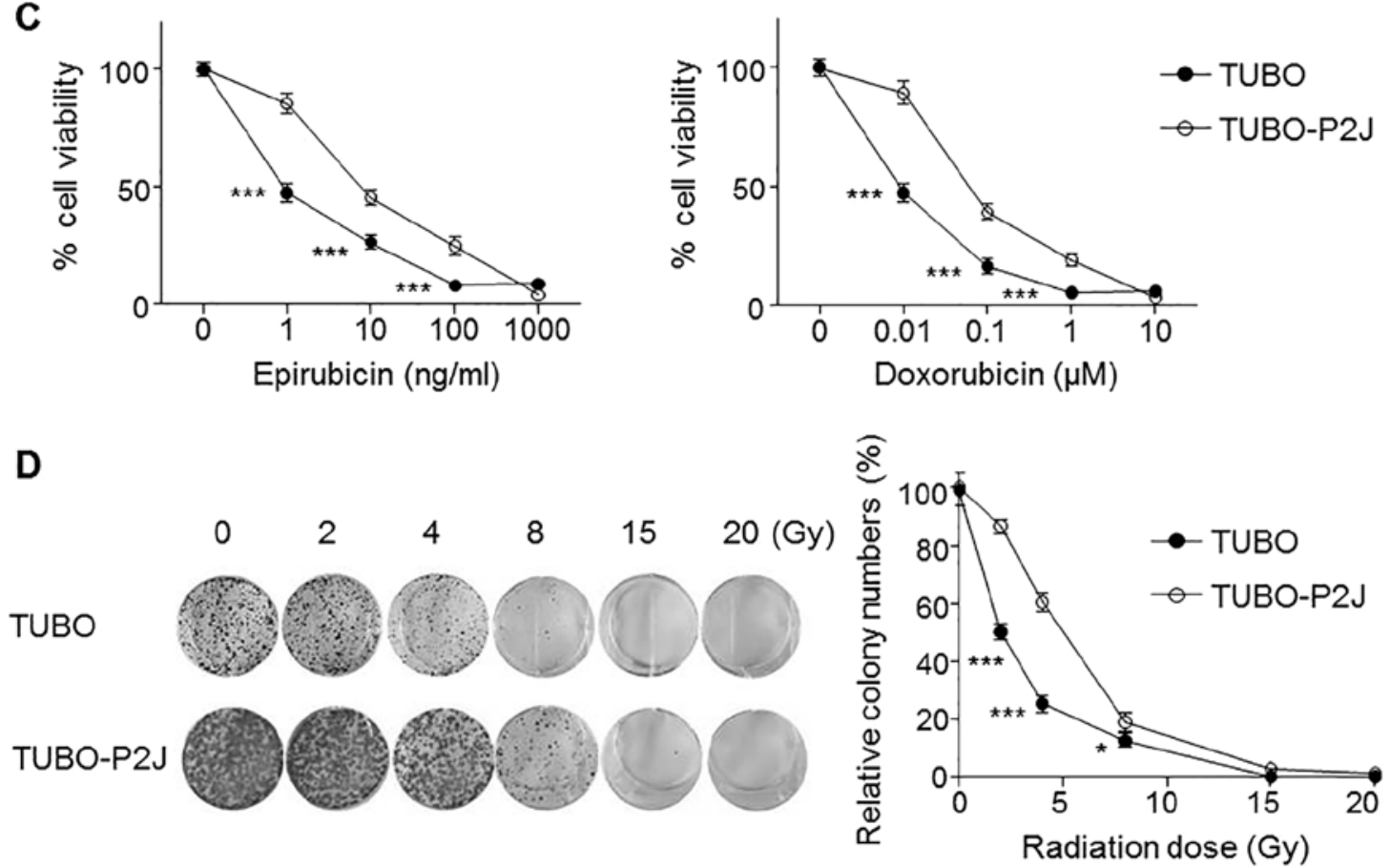

Figure 1. Mesenchymal TUBO-P2J cells show cancer stem cell-like properties and increased resistance to chemotherapy and radiotherapy compared to parental epithelial TUBO cells. (A) CD44 and CD24 expression levels of TUBO and TUBO-P2J cells were quantified by flow cytometry. (B) Soft agar colony-formation assays were conducted by seeding TUBO and TUBO-P2J cells on $0.5 \%$ agarose in 6 -well plates. After 12 days of culturing, colonies were stained with crystal violet and counted under a microscope. The data shown are representative of three independent experiments (mean \pm SD). (C) Chemosusceptibility was determined by a Tox -6 assay, and the data are presented as the mean \pm SD. (D) Radiation susceptibility was determined by seeding irradiated cells in 6-well plates at a 10-fold-dilution. After $72 \mathrm{~h}$, the cells were stained with crystal violet and counted under a microscope. The data are presented as the mean \pm SD. ${ }^{*} \mathrm{p}<0.01 ;{ }^{* * *} \mathrm{p}<0.001$. 
(RapidArc ${ }^{\circledR}$; Varian Medical Systems Inc., Palo Alto, CA, USA), cells were diluted 10 -fold and seeded in 6-well plates. After $72 \mathrm{~h}$, the cells were stained with $0.5 \%$ crystal violet for 10 min and fixed with $4 \%$ paraformaldehyde. Colonies were counted under a microscope. For in vivo radiation treatment, TUBO-P2J/NC $\left(1.5 \times 10^{5}\right)$ and TUBO-P2J/shSnail $\left(2 \times 10^{5}\right)$ cells were implanted subcutaneously and tumour tissues were locally irradiated at 15 -Gy with an X-ray generator under anaesthesia (ketamine $90 \mathrm{mg} / \mathrm{kg}$ and xylazine $10 \mathrm{mg} / \mathrm{kg}$ ).

In vivo animal study. All of the procedures involving animals were approved by the INJE University College of Medicine Institutional Animal Care and Use Committee. All of the mice (BALB/C, 6-8 weeks of age) used in this study were purchased from Orient Bio (Taejun, Korea). To evaluate the tumour formation ability and growth, $1 \times 10^{5}$ or $1 \times 10^{4}$ cells were subcutaneously implanted on the backside of the mice. Tumour development was followed every day, and tumour sizes were measured two times per week. Tumour volumes were measured along three orthogonal axes (x, y, and $\mathrm{z})$ and calculated as tumour volume $=(x y z) / 2$. To analyse experimental metastasis, $2 \times 10^{4}$ cells were intravenously injected through the tail vein and lung tissues were collected at day 19 .

Statistics. Differences between groups were analysed using an unpaired t-test. Error bars represent \pm SD. All statistical analyses were conducted using Graph-Pad Prism Version 4.0 (GraphPad Software). Unless specified, statistically significant differences of $\mathrm{p}<0.05,0.01$, and 0.001 are noted.

\section{Results}

TUBO-P2J displays a cancer stem cell phenotype and resistance to chemotherapy and radiotherapy. Previously we reported on the the highly metastatic mouse breast cancer cell line TUBO-P2J, derived from the non-metastatic TUBO cell line (10). TUBO cells were morphologically epithelial in nature. In contrast, TUBO-P2J cells were spindle-shaped mesenchymal-type cells. TUBO-P2J cells showed a gain of Snail and loss of E-cadherin. In flow cytometry analysis and soft agar colony assays, the TUBO-P2J cell line was characterized as stem cell-like with a prominent $\mathrm{CD} 44^{\text {high }}$ population (Fig. 1A) and 5 times higher colony number (Fig. 1B) than TUBO cells. Chemo and radiation susceptibility tests revealed that TUBO-P2J cells were more resistant to chemotherapy and radiation therapy (Fig. 1C and D). Snail expression levels were associated with poor prognosis phenotypes, including metastatic potential, soft agar colony formation capacity and resistance to chemotherapy and radiotherapy.

Before Snail silencing, we compared the migration potentials of TUBO-P2J cell line with other breast cancer cell lines, such as MCF7, MDA-MD-231, HCC70, 4T1, and original TUBO cell lines (Fig. 2A). The migration activity of TUBO-P2J was higher than that of human breast cancer MDA-MB-231, which is a well-known cell line with a highly metastatic potential. TUBO-P2J cell line also showed the highest level of snail expression among the tested breast cancer cell lines (Fig. 2B). In addition, TUBO-P2J cell line did not express estrogen receptor $\alpha 1$ (ESR1) similarly to MDA-MB-231, triple-negative
A
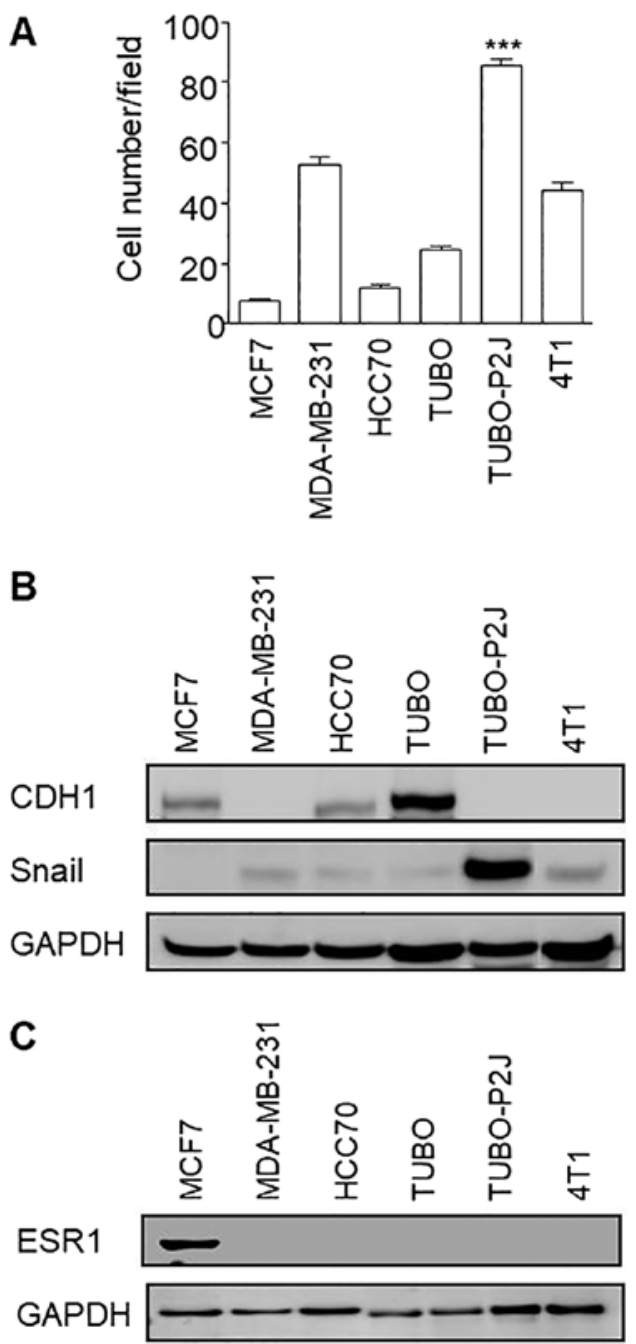

Figure 2. TUBO-P2J cell line has high metastatic potential via Snail expression. (A) Transwell migration assay at $5 \times 10^{4}$ cells of MCF7, MDA-MB-231, HCC70, TUBO, TUBO-P2J and 4T1 cells added to the upper chamber and incubated for $6 \mathrm{~h}$. Migrated cells were counted from ten randomly selected fields under $x 40$ magnification and averages were calculated. The data shown are representative of three independent experiments. The data are presented as the mean \pm SD. ${ }^{*} \mathrm{p}<0.01 ;{ }^{* * *} \mathrm{p}<0.001$. B and C) Total protein was extracted at stable states of human and mouse breast cancer cells and CDH1, Snail and ESR1 were detected. The protein levels were normalized to the level of GAPDH in each sample.

breast cancer cell line (Fig. 2C). Even though TUBO-P2J is a mouse tumour cell line, it is derived spontaneously in vivo and could be exactly compared with parent TUBO cells which showed less malignant phenotypes, including metastatic potential, stemness, chemoresistance and radioresistance (10). Based on these data, we chose the TUBO-P2J cell line for Snail silencing experiments with efficient lentiviral transfection system.

Downregulation of Snail does not induce mesenchymalepithelial transition in TUBO-P2J cells. To examine the role of Snail in maintaining the mesenchymal phenotype and the suppression of E-cadherin, we produced a stable Snail knockdown TUBO-P2J cell line (TUBO-P2J/shSnail) by lentiviral transfection. Two independent stable shSnailexpressing clones (Sh1 and Sh3, one clone from each construct) and a stable negative control shRNA clone (NC) 

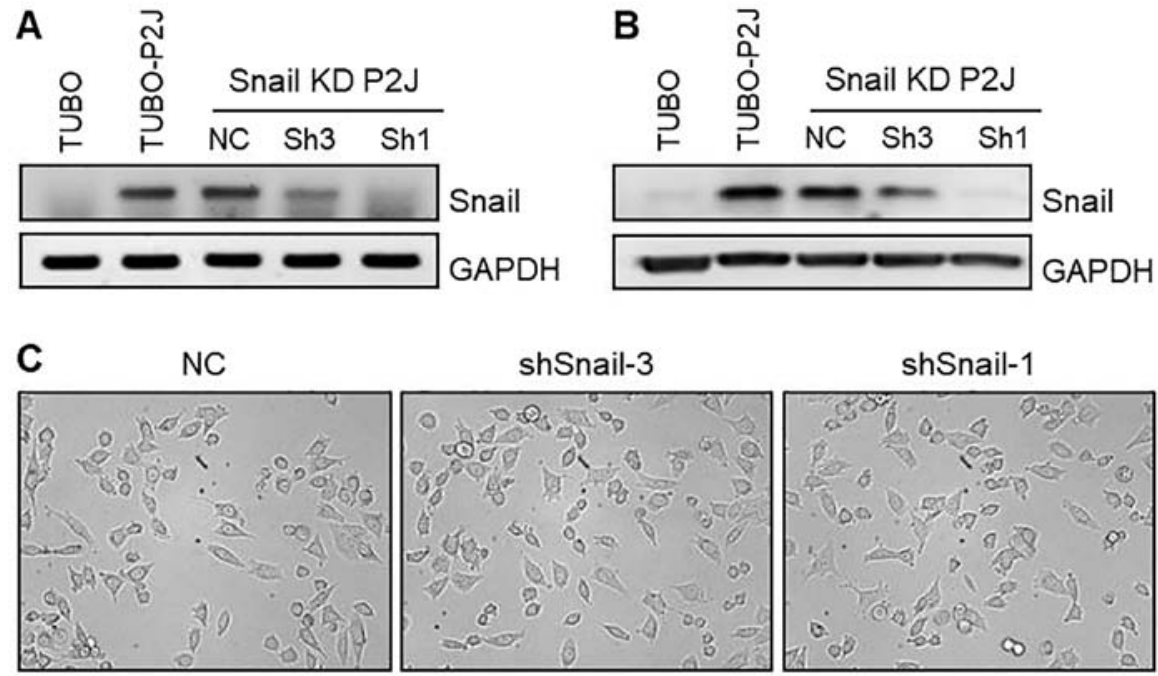

$(\times 200)$
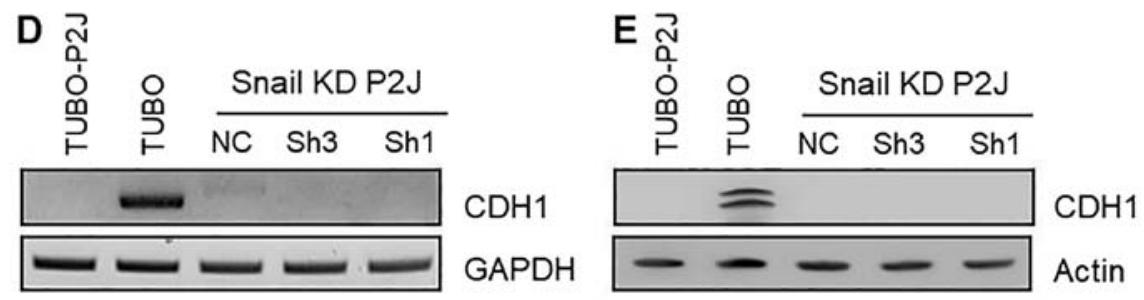

Figure 3. Silencing Snail with shRNA does not induce morphological changes or restore E-cadherin expression. Snail mRNA (A) and protein (B) expression levels of stable shRNA expressing (Sh3, Sh1) and negative control shRNA-expressing (NC) cells. (C) Bright field micrographs of Sh3, Sh1, and NC cells. E-cadherin mRNA (D) and protein (E) expression levels of Sh3, Sh1, and NC cells. GAPDH or actin were used as internal controls.

were analysed for Snail mRNA expression. Sh1 and Sh3 showed $50-90 \%$ downregulation of Snail mRNA, whereas the control clone did not exhibit any significant reduction in Snail mRNA (Fig. 3A). Knockdown of Snail in TUBO-P2J cells was confirmed with western blot analysis (Fig. 3B). Snail protein levels were correlated with mRNA levels. The morphology of Snail knockdown cells (Sh1 and Sh3) and control cells (NC) was not different, as all of the cells showed a spindle-shaped mesenchymal phenotype (Fig. 3C). Next, we evaluated the expression of E-cadherin with RT-PCR and western blot analysis (Fig. 3D and E). In TUBO-P2J cells, Snail knockdown did not induce transcription of E-cadherin. Based on Snail expression levels, we chose the Sh1 clone as TUBO-P2J/shSnail for further experiments.

E-cadherin expression was controlled by DNA methylation, histone deacetylation, and Snail in TUBO-P2J cells. Lim et al (12) reported that Snail represses the transcription of E-cadherin through binding to E-boxes of this gene and inducing DNA methylation of its promoter by recruiting HDAC1 and DNMT. To evaluate the reason that E-cadherin was not expressed in TUBO-P2J/shSnail cells, cells were treated with 5-aza-dC and/or SAHA. By the third day of 5-azaC treatment, E-cadherin mRNA was detected in all TUBO-P2J cells regardless of Snail knockdown (Fig. 4A). However, E-cadherin protein was detected only in shSnail cells by treatment of 5-azaC and SAHA for 7 days (Fig. 4B). These data suggest that Snail might suppress the expression of E-cadherin through a translation step co-operating with HDAC in TUBO-P2J cells.
Snail increases metastatic potential in TUBO-P2J cells. Because processes of EMT have been linked with metastasis, we next evaluated the metastatic potential of PLKO and Sh1 cells. By silencing Snail, migration was significantly decreased from $195.3 \pm 7.5$ (mean $\pm \mathrm{SE}$ ) cells/fields to $86.7 \pm 4.7$ cells/fields (56\% decrease) (Fig. 5A) and invasion was decreased even more from $127.1 \pm 47.6$ cells/fields to $10.7 \pm 2.7$ cells/fields (93\% decrease) (Fig. 5B). An in vivo metastasis assay using tail vein injection also showed that lung colony numbers of shSnail cells were reduced to one-third of that of control cells (Fig. 5C). These data suggest that inactivation of Snail could reduce the metastatic potentials of breast cancer cells without driving MET.

Snail maintains cancer stem cell-like properties and in vivo tumourigenicity of TUBO-P2J cells. As Snail is expressed in CD $44^{\text {high }}$ compared to CD $44^{\text {low }}$ cells, we examined whether Snail could affect the expression of CD44, a breast cancer stem cell marker. Snail silenced TUBO-P2J cells showed a slight decrease in the CD44 ${ }^{\text {high }}$ population $(37.5 \%)$ compared to their control counterparts (52.9\%) (Fig. 6A). Aldehyde dehydrogenase (ALDH) activity was also reduced significantly by Snail silencing (Fig. 6B). Next, we used a soft agar colonization assay to evaluate the role of Snail in anchorage-independent growth. Data showed that the sizes of the colonies were not different with Snail silencing, but the numbers were significantly reduced by $40 \%$ compared to that of control cells (Fig. 6C). In vivo tumourigenicity was tested with subcutaneous implantation of TUBO-P2J, TUBO-P2J/NC, and TUBO-P2J/shSnail $\left(1 \times 10^{4}\right.$ or $1 \times 10^{5}$ cells/mouse). In all of the mice implanted 
A Day 3

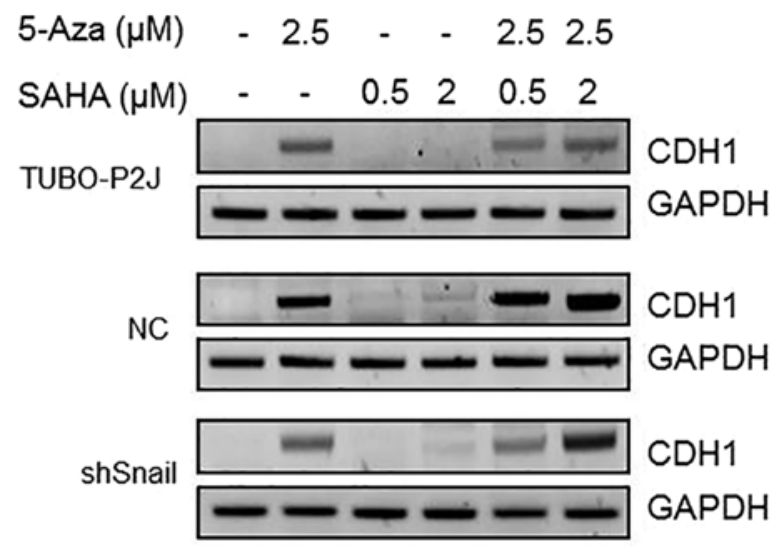

B Day 3

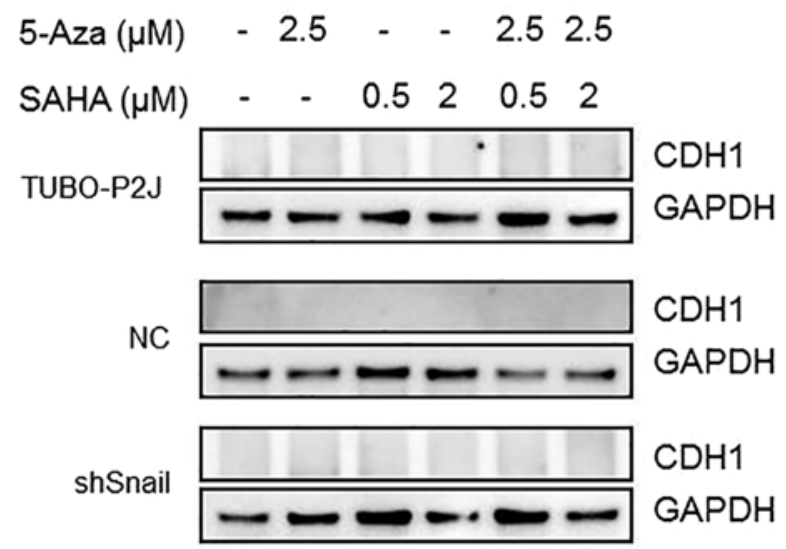

\section{Day 7}

$\begin{array}{lllllll}\text { 5-Aza }(\mu \mathrm{M}) & - & 2.5 & - & - & 2.5 & 2.5 \\ \text { SAHA }(\mu \mathrm{M}) & - & - & 0.5 & 2 & 0.5 & 2\end{array}$

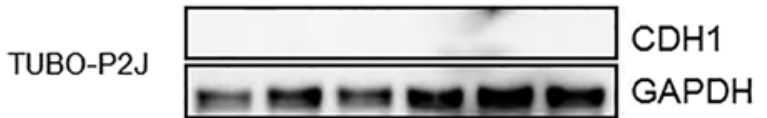

NC

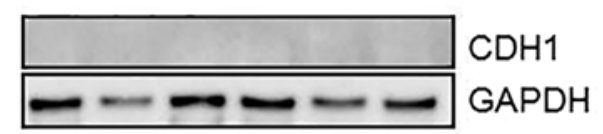

shSnail

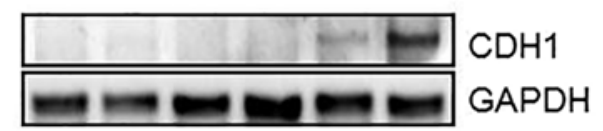

Figure 4. E-cadherin expression is restored by inhibition of DNMT and HDAC in TUBO-P2J/shSnail cells. TUBO-P2J, TUBO-P2J/NC, and TUBO-P2J/shSnail cells were treated with 5-aza-dC $(2.5 \mu \mathrm{M})$ and/or SAHA $(0.5$ or $2 \mu \mathrm{M})$. (A) E-cadherin mRNA expression was measured with reverse transcription (RT)-PCR analysis at day 3. (B) E-cadherin protein levels were measured with western blot analysis at days 3 and 7 .

with parent TUBO-P2J and TUBO-P2J/NC cells, tumours developed at day 14, but tumours developed in two mice out of seven at 30 days when $1 \times 10^{4}$ of TUBO-P2J/shSnail cells were implanted (Table II). Moreover, a decrease in tumour size was observed in tumours established with Snail silenced cells compared with those from parent and control cells (Fig. 6D).
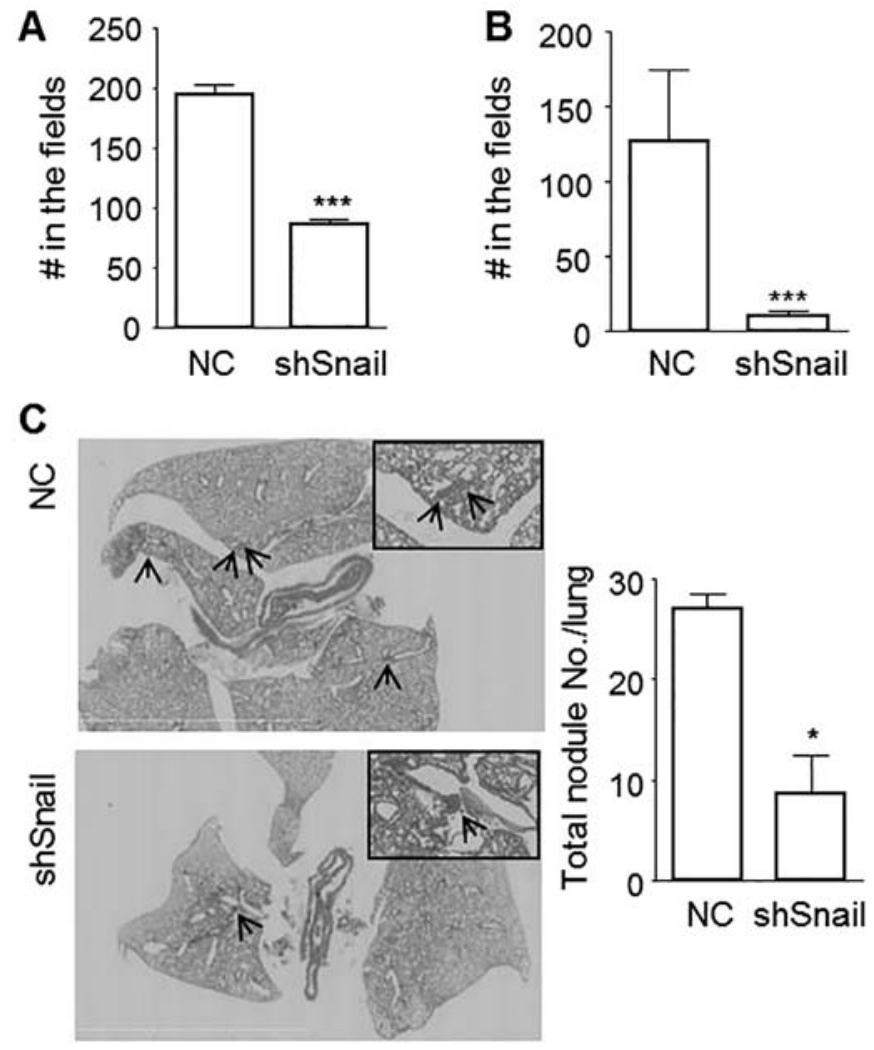

Figure 5. Silencing Snail reduces metastatic potential. (A) Migration assay. TUBO-P2J/NC and TUBO-P2J/shSnail cells $\left(5 \times 10^{4}\right)$ were added to the top of Transwell plates and incubated for $4 \mathrm{~h}$. Migrated cells were counted from 10 randomly selected fields under $\mathrm{x} 40$ magnification, and the averages were calculated. The data shown are representative of three independent experiments (mean $\pm \mathrm{SD}$ ). (B) Invasion assay. TUBO-P2J/NC and TUBO-P2J/ shSnail cells $\left(1 \times 10^{5}\right)$ were added to Matrigel-coated Transwell plates and incubated for 3 days. Invaded cells were counted from 10 randomly selected fields under $\mathrm{x} 40$ magnification, and the averages were calculated. The data shown are representative of three independent experiments (mean $\pm \mathrm{SD}$ ). (C) Experimental metastasis assay. TUBO-P2J/NC and TUBO-P2J/shSnail cells $\left(1 \times 10^{5}\right)$ cells were injected intravenously through the tail vein. Lung tissues were collected at day 19 and stained with H\&E. Colonies in the lung were counted under a microscope. The data were collected from 4 mice for each cell line (mean $\pm \mathrm{SD}) .{ }^{*} \mathrm{p}<0.01 ;{ }^{* * *} \mathrm{p}<0.001$.

Table II. Incidence of tumour growth.

\begin{tabular}{lll}
\hline & \multicolumn{2}{c}{ Cell numbers injected } \\
\cline { 2 - 3 } Tumour cells & $10^{5}$ & $10^{4}$ \\
\hline TUBO-P2J & $8 / 8$ & $6 / 6$ \\
NC & $7 / 7$ & $6 / 6$ \\
shSnail & $7 / 7$ & $2 / 7$ \\
\hline
\end{tabular}

These in vitro and in vivo data demonstrate that Snail has an important role in the maintenance of breast cancer stem cell properties.

Snail increases chemoresistance but not radioresistance. As Snail is expressed in chemo- and radioresistant cells, TUBO-P2J, we examined whether Snail could induce chemo- and radioresistance. Our data revealed that silencing 
A
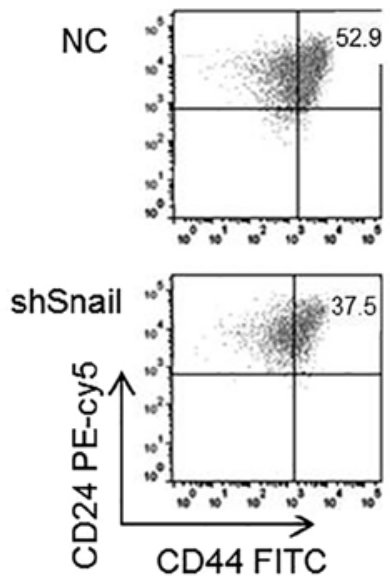

B
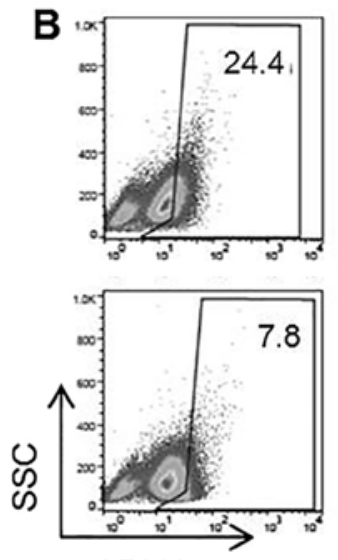

ADLH
C

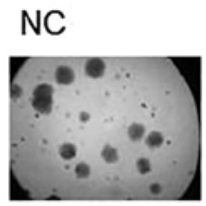

shSnail

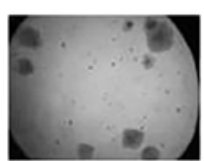

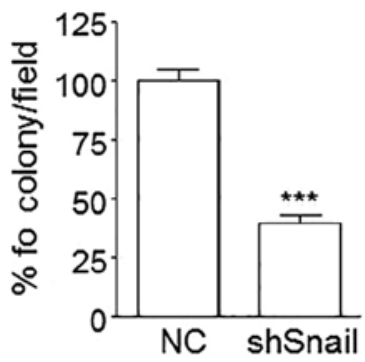

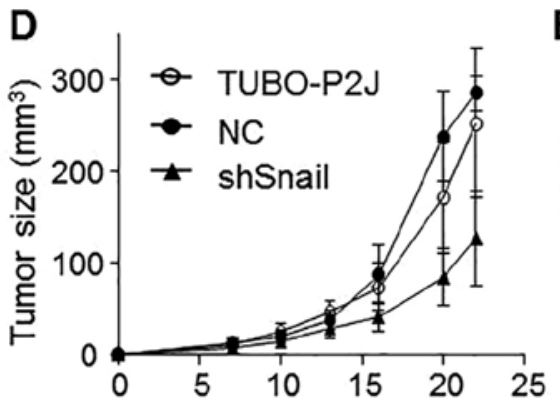

Day after tumor implantation
E

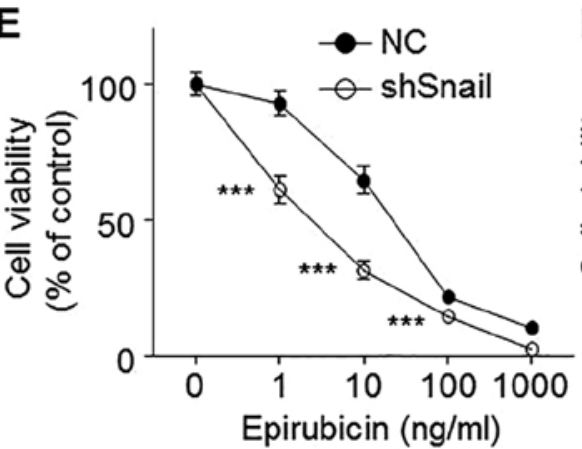

F

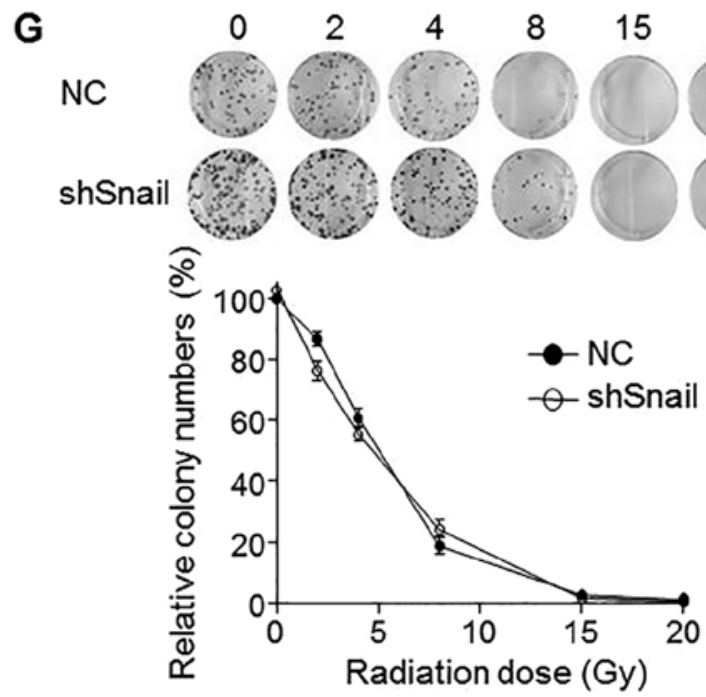

H

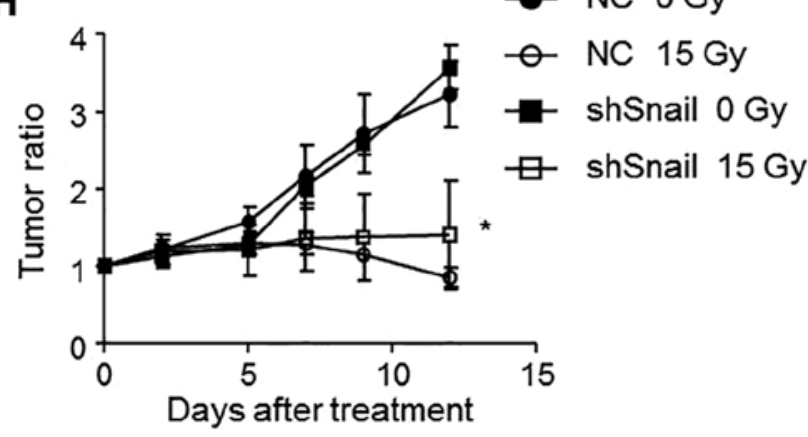

Figure 6. Silencing Snail induces downregulation of tumourigenicity and chemoresistance but not conneted radioresistance. (A) CD44 and CD24 expression levels of TUBO-P2J/NC and TUBO-P2J/shSnail cells were quantified by flow cytometry. (B) ALDH1 activity of TUBO-P2J/NC and TUBO-P2J/shSnail cells. Cells were treated with an Aldefluor substrate in the presence or absence of ALDH inhibitors DEAB. After treatment, the samples were analysed by flow cytometry for the presence of ALDH ${ }^{\text {high }}$ cells. (C) A soft agar colony-formation assay for TUBO-P2J/NC and TUBO-P2J/shSnail cells. (D) Tumour growth curves: $1 \times 10^{5}$ tumour cells were implanted subcutaneously on the backs of mice. Tumour growth was monitored twice per week, and the data are presented as the mean tumour size $\pm \mathrm{SD}$ of 4 tumours per group. The data shown are representative of two independent experiments. (E and F) TUBO-P2J/NC and TUBO-P2J/shSnail cells were treated with epirubicin (E) and doxorubicin (F) for 72 h. Cell viability was measured with a Tox-6 assay, and the data are presented as mean \pm SD. (G) TUBO-P2J/NC and TUBO-P2J/shSnail cells were irradiated with the indicated dose and seeded in 6 -well plates at a 10 -fold-dilution. After $72 \mathrm{~h}$, the cells were stained with crystal violet and counted under a microscope. (H) Tumour-bearing mice were irradiated locally with $15 \mathrm{~Gy}$, and tumour sizes were measured twice per week. The data are presented as the relative tumour size compared to the tumour size when treatment was started (mean $\pm \mathrm{SD}){ }^{*} \mathrm{p}<0.01 ;{ }^{* * *} \mathrm{p}<0.001$.

Snail increased the susceptibility to epirubicin and doxorubicin (Fig. 6E and $\mathrm{F}$ ); however, susceptibility to radiation therapy was not affected by Snail silencing (Fig. 6G and H). These results suggested that Snail silencing might be a good strategy to overcome chemoresistance.
The expression of SLUG and Vimentin and the $\beta$-catenin signaling are not changed by Snail silencing. To test whether the phenotype changes in Snail silent cells were mediated by other EMT related genes, we evaluated mRNA levels of EMT related genes such as SLUG (Snail2), vimentin, 

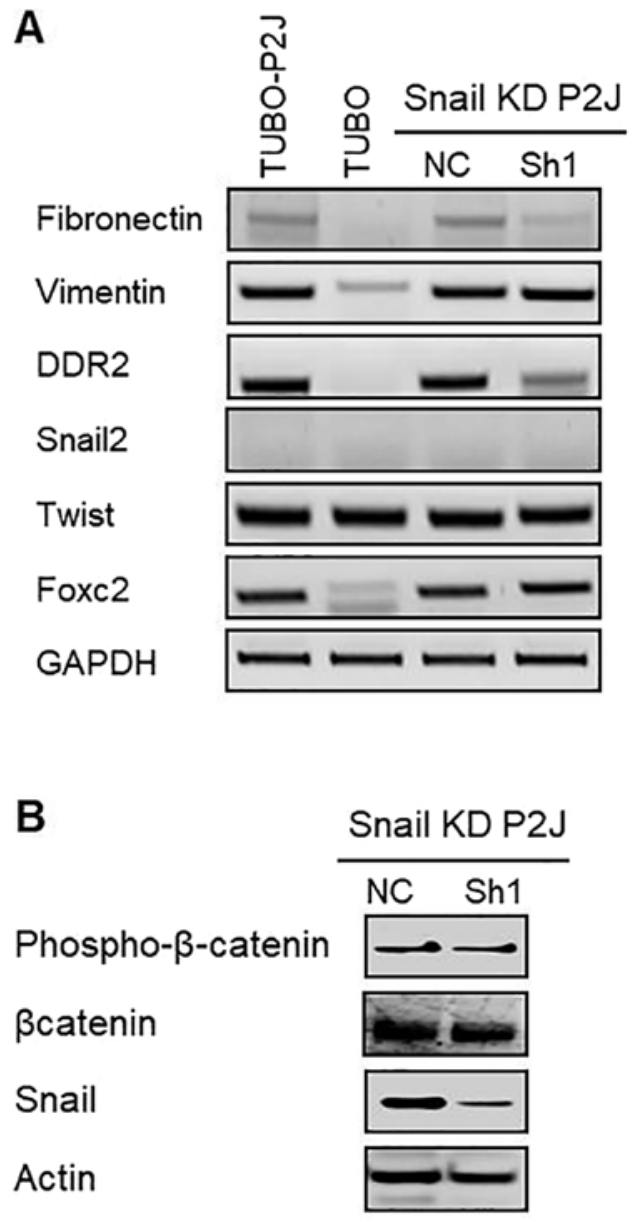

Figure 7. The expression of Snail 2 and vimentin and the $\beta$-catenin signaling were not changed by Snail silencing. (A) mRNA expressions of EMT related genes in Snail silent cells were evaluated with reverse transcription (RT)-PCR analysis. (B) $\beta$-catenin singaling was evaluated with western blot analysis for non-phospho and total $\beta$-catenin protein in total protein.

fibrinectin, Twist, DDR2, and FoxC2 (Fig. 7A). Data revealed that silencing of Snail reduced the expressions of fibronectin and DDR2 but did not influence on the expressions of other EMT related genes including vimentin, SLUG, and Twist. In addition, silencing of Snail did not change the activation status of $\beta$-catenin (Fig. 7B). These data suggested that the malignant phenotypes of TUBO-P2J cell lines which are increased stemness, metastasis, and chemoresistance are mediated by the increased expression of Snail and the role of Snail in this model was not linked with the expression of vimentin and SLUG and Wnt/ $\beta$-catenin signaling pathway.

\section{Discussion}

This study demonstrates that Snail, one of the master EMT genes in breast cancer cells, maintains metastatic potential, clonogenicity, and drug resistance without restoration of E-cadherin expression or morphological changes. In addition, our results also revealed that Snail is required for tumour initiation and growth in vivo. Our data suggested that inhibition of Snail activity could be a good strategy to inhibit tumour metastasis and to enhance the biological effects of anticancer agents.
Loss of E-cadherin is a hallmark of the EMT process. E-cadherin expression is controlled by various transcription factors, Snail genes, Twist, Zeb genes and E47 $(1,4)$. Snail was discovered first and is the most important transcriptional repressor of E-cadherin (6). Snail binds to the E-box of the E-cadherin promoter through $\mathrm{C}_{2} \mathrm{H}_{2}$-type zinc fingers and represses transcription (13). Many reports have demonstrated that silencing Snail can restore E-cadherin expression in various cancer cells including breast cancer (14-20). In TUBO-P2J cells, however, silencing Snail did not restore the expression of E-cadherin. This finding might be because: i) Snail sufficiently represses E-cadherin during the initial stages of EMT while subsequent maintenance may require cooperation of other transcription factors (21), or ii) Snail can play a role as a mediator of epigenetic changes through recruiting histone deacetylases (HDAC) and DNA methyltransferase (DNMT) to the E-cadherin promoter $(12,14)$. Though we cannot rule out the possibility that other transcription factors repress the expression of E-cadherin, epigenetic changes are a main regulator for E-cadherin expression in TUBO-P2J cells. Interestingly, the transcription of E-cadherin was restored by 5 -aza-dC treatment within 3 days regardless of the presence of Snail, but the E-cadherin protein was detected only in TUBO-P2J/shSnail cells treated with both 5 -aza-dC and SAHA at day 7. These data suggested that Snail may regulate E-cadherin expression at post-transcriptional steps with HDAC. Further studies to define the exact mechanisms of Snail in the post-transcriptional regulation of E-cadherin and which types of HDAC participate would be required.

In breast cancer, Snail expression has been detected at the invasive area, coincidently with E-cadherin downregulation, and has been associated with lymph node metastasis and recurrence $(17,22)$. Previous studies demonstrated that Snail participates in the EMT-MET process, tumour metastasis, cancer stemness, recurrence, and resistance to chemotherapy and radiotherapy $(6-9,23)$. In these studies, however, the expression of Snail was linked with E-cadherin expression and morphological changes, such as EMT or MET. Therefore, it is not clear whether these poor prognostic potentials are induced by Snail-induced EMT or by Snail expression itself. In the metastatic potential, silencing Snail significantly reduced in vitro migration and invasion and in vivo lung colonization. These data revealed that Snail plays a role as a central regulator of metastasis. For the breast cancer stem cell markers, CD44 expression levels and ALDH activity were also decreased by Snail silencing. In human breast cancer, various markers, such as CD24,CD44,CD133, CD166 and ALDH1, were identified as cancer stem cell markers, as cells with the phenotype $\mathrm{CD}_{4} 4^{+} /$ $\mathrm{CD} 24^{\text {low/ }}$ and/or $\mathrm{ALDH} 1^{+}$are most consistently associated with stem-like characteristics (24-28). In our study, CD24 expression levels were not different between epithelial non-metastatic TUBO cells and mesenchymal metastatic TUBO-P2J cells and were not linked with colony formation ability. In these mouse breast cancer cell lines, CD44 expression levels were linked with colony formation ability and in vivo tumour formation and growth and were regulated by Snail expression. ALDH1 activities in TUBO and TUBO-P2J cells were similar (data not shown); however, silencing Snail reduced ALDH1 activity in TUBO-P2J cells. Though the reasons that CD24 expression was not linked with cancer stemness remain 
unknown, it is clear that CD44 expression levels are closely related with cancer stemness and are regulated by Snail expression. Regarding resistance to chemo-drugs, while we tested only two anthracyline drugs, our results are consistent with prior studies that suggested that EMT is associated with an increased chemoresistance of cancer cells (8). However, there are some controversies regarding the link between EMT or Snail and chemoresistance. Mezencev et al (23) reported that Snail-induced EMT in MCF7 cells induced resistance to gemcitabine and mitomycin $\mathrm{C}$, but increased sensitivity to doxorubicin, methotrexate, cisplatin, and 5-fluorouracil. In our previous study, we showed that mesenchymal TUBO-P2J cells were less sensitive to 12 breast cancer chemo-drugs than parental epithelial TUBO cells (10). It is still difficult to generalize whether Snail or EMT increase the resistance of breast cancer cells to most chemo-drugs, but our results suggest the possibility that Snail inhibition can reduce the resistance to some chemo-drugs. A number of studies have reported that EMT and cancer stem cell-like properties are also associated with radioresistance in breast cancer cells $(9,29-31)$. However, it is not clear whether Snail regulates radioresistance in breast cancer. Mezencev et al (23) showed that mesenchymal MCF7-Snail cells are more sensitive to radiation that their parental cells. Zhang et al (32) demonstrated that radioresistance can be induced and maintained by ZEB1, not by Twist and Snail, using gene expression and silencing techniques. Our results also showed that silencing Snail without E-cadherin restoration or morphological changes did not increase the sensitivity to radiation. Considering cancer stem-like properties were decreased by Snail silencing and there was no detection of ZEB1 in TUBO-P2J cells (10), radioresistance might be regulated by unknown factors in TUBO-P2J cells.

Based on this study, Snail is thought to be a critical element in the machinery that maintains the metastatic potential, stemlike properties and chemoresistance in mesenchymal breast cancer. In addition, Snail inhibition would be a good target for breast cancer treatment.

\section{Acknowledgements}

This study was supported by the National Research Foundation of Korea (NRF) grant funded by the Korea government (MSIP) (NRF-2015R1A2A2A01008394).

\section{References}

1. Thiery JP, Acloque H, Huang RY and Nieto MA: Epithelialmesenchymal transitions in development and disease. Cell 139: 871-890, 2009.

2. Scheel C and Weinberg RA: Cancer stem cells and epithelialmesenchymal transition: Concepts and molecular links. Semin Cancer Biol 22: 396-403, 2012.

3. Tiwari N, Gheldof A, Tatari M and Christofori G: EMT as the ultimate survival mechanism of cancer cells. Semin Cancer Biol 22: 194-207, 2012.

4. Lamouille S, Xu J and Derynck R: Molecular mechanisms of epithelial-mesenchymal transition. Nat Rev Mol Cell Biol 15: 178-196, 2014.

5. de Herreros AG, Peiró S, Nassour M and Savagner P: Snail family regulation and epithelial mesenchymal transitions in breast cancer progression. J Mammary Gland Biol Neoplasia 15: $135-147,2010$.

6. Wu Y and Zhou BP: Snail: More than EMT. Cell Adhes Migr 4: 199-203, 2010.
7. Moody SE, Perez D, Pan TC, Sarkisian CJ, Portocarrero CP, Sterner CJ, Notorfrancesco KL, Cardiff RD and Chodosh LA: The transcriptional repressor Snail promotes mammary tumor recurrence. Cancer Cell 8: 197-209, 2005.

8. Foroni C, Broggini M, Generali D and Damia G: Epithelialmesenchymal transition and breast cancer: Role, molecular mechanisms and clinical impact. Cancer Treat Rev 38: 689-697, 2012.

9. Theys J, Jutten B, Habets R, Paesmans K, Groot AJ, Lambin P, Wouters BG, Lammering G and Vooijs M: E-cadherin loss associated with EMT promotes radioresistance in human tumor cells. Radiother Oncol 99: 392-397, 2011.

10. Song H, Kim TO, Ma SY, Park JH, Choi JH, Kim JH, Kang MS, Bae SK, Kim KH, Kim TH, et al: Intratumoral heterogeneity impacts the response to anti-neu antibody therapy. BMC Cancer 14: 647, 2014.

11. Ma SY, Song H, Park JH, Choi JH, Kim JH, Kim KH, Park S, Park DH, Kang MS, Kwak M, et al: Addition of anti-neu antibody to local irradiation can improve tumor-bearing BALB/c mouse survival through immune-mediated mechanisms. Radiat Res 183: 271-278, 2015.

12. Lim SO, Gu JM, Kim MS, Kim HS, Park YN, Park CK, Cho JW, Park YM and Jung G. Epigenetic changes induced by reactive oxygen species in hepatocellular carcinoma: Methylation of the E-cadherin promoter. Gastroenterology 135: 2128-2140, 2140.e1-8, 2008.

13. Nieto MA: The snail superfamily of zinc-finger transcription factors. Nat Rev Mol Cell Biol 3: 155-166, 2002.

14. Dong C, Wu Y, Yao J, Wang Y, Yu Y, Rychahou PG, Evers BM and Zhou BP: G9a interacts with Snail and is critical for Snailmediated E-cadherin repression in human breast cancer. J Clin Invest 122: 1469-1486, 2012.

15. Harney AS, Meade TJ and LaBonne C: Targeted inactivation of Snail family EMT regulatory factors by a Co(III)-Ebox conjugate. PLoS One 7: e32318, 2012.

16. Zhou W, Lv R, Qi W, Wu D, Xu Y, Liu W, Mou Y and Wang L: Snail contributes to the maintenance of stem cell-like phenotype cells in human pancreatic cancer. PLoS One 9: e87409, 2014.

17. Olmeda D, Moreno-Bueno G, Flores JM, Fabra A, Portillo F and Cano A: SNAI1 is required for tumor growth and lymph node metastasis of human breast carcinoma MDA-MB-231 cells. Cancer Res 67: 11721-11731, 2007.

18. Olmeda D, Montes A, Moreno-Bueno G, Flores JM, Portillo F and Cano A: Snail and Snai2 collaborate on tumor growth and metastasis properties of mouse skin carcinoma cell lines. Oncogene 27: 4690-4701, 2008.

19. Zhang A, Chen G, Meng L, Wang Q, Hu W, Xi L, Gao Q, Wang S, Zhou J, Xu G, et al: Antisense-Snail transfer inhibits tumor metastasis by inducing E-cadherin expression. Anticancer Res 28A: 621-628, 2008.

20. Smith BN, Burton LJ, Henderson V, Randle DD, Morton DJ, Smith BA, Taliaferro-Smith L, Nagappan P, Yates C, Zayzafoon M, et al: Snail promotes epithelial mesenchymal transition in breast cancer cells in part via activation of nuclear ERK2. PLoS One 9: e104987, 2014.

21. Lee $\mathrm{K}$ and Nelson CM: New insights into the regulation of epithelial-mesenchymal transition and tissue fibrosis. Int Rev Cell Mol Biol 294: 171-221, 2012.

22. Blanco MJ, Moreno-Bueno G, Sarrio D, Locascio A, Cano A, Palacios J and Nieto MA: Correlation of Snail expression with histological grade and lymph node status in breast carcinomas. Oncogene 21: 3241-3246, 2002.

23. Mezencev R, Matyunina LV, Jabbari N and McDonald JF: Snail-induced epithelial-to-mesenchymal transition of MCF-7 breast cancer cells: Systems analysis of molecular changes and their effect on radiation and drug sensitivity. BMC Cancer 16: 236, 2016

24. Abraham BK, Fritz P, McClellan M, Hauptvogel P, Athelogou M and Brauch $\mathrm{H}$ : Prevalence of $\mathrm{CD} 44^{+} / \mathrm{CD} 24 /$ low cells in breast cancer may not be associated with clinical outcome but may favor distant metastasis. Clin Cancer Res 11: 1154-1159, 2005.

25. Ponti D, Costa A, Zaffaroni N, Pratesi G, Petrangolini G, Coradini D, Pilotti S, Pierotti MA and Daidone MG: Isolation and in vitro propagation of tumorigenic breast cancer cells with stem/progenitor cell properties. Cancer Res 65: 5506-5511, 2005.

26. Ginestier C, Hur MH, Charafe-Jauffret E, Monville F, Dutcher J, Brown M, Jacquemier J, Viens P, Kleer CG, Liu S, et al: ALDH1 is a marker of normal and malignant human mammary stem cells and a predictor of poor clinical outcome. Cell Stem Cell 1: 555-567, 2007. 
27. Jaggupilli A and Elkord E: Significance of CD44 and CD24 as cancer stem cell markers: An enduring ambiguity. Clin Dev Immunol 2012: 708036, 2012.

28. de Beça FF, Caetano P, Gerhard R, Alvarenga CA, Gomes M, Paredes J and Schmitt F: Cancer stem cells markers CD44, CD24 and ALDH1 in breast cancer special histological types. J Clin Pathol 66: 187-191, 2013.

29. Santisteban M, Reiman JM, Asiedu MK, Behrens MD, Nassar A Kalli KR, Haluska P, Ingle JN, Hartmann LC, Manjili MH, et al: Immune-induced epithelial to mesenchymal transition in vivo generates breast cancer stem cells. Cancer Res 69: 2887-2895, 2009.
30. Pajonk F, Vlashi E and McBride WH: Radiation resistance of cancer stem cells: The 4 R's of radiobiology revisited. Stem Cells 28: 639-648, 2010.

31. Yin $\mathrm{H}$ and Glass $\mathrm{J}$ : The phenotypic radiation resistance of $\mathrm{CD} 44^{+} / \mathrm{CD} 24$ (-or low) breast cancer cells is mediated through the enhanced activation of ATM signaling. PLoS One 6: e24080, 2011.

32. Zhang P, Wei Y, Wang L, Debeb BG, Yuan Y, Zhang J, Yuan J, Wang M, Chen D, Sun Y, et al: ATM-mediated stabilization of ZEB1 promotes DNA damage response and radioresistance through CHK1. Nat Cell Biol 16: 864-875, 2014. 